\title{
QUALIDADE DA HIGIENIZAÇÃO DAS MÃOS DE PROFISSIONAIS AT UANTES EM UNIDADES BÁSICAS DE SAÚDE
}

\author{
Lindsay LOCKS ${ }^{a}$, J osimari T elino LACERDA ${ }^{b}$, Elonir GOM ES ${ }^{c}$, A na Claudina Prudêncio SE R RAT INE ${ }^{c}$
}

\section{RESUM 0}

0 estudo teve por objetivo avaliar a qualidade de higienização das mãos de profissionais. A pesquisa foi conduzida em todas as U nidades Básicas de Saúde de dez municípios da Região Sul de Santa Catarina em 2008. Foi Estudo epidemiológico transversal, desenvolvido com a observação de 369 profissionais da saúde. Real izou-se o teste Quiquadrado para verificar associação de variáveis sócio-demog ráficas e formação com o desempenho dos profissionais. D urante os procedimentos cirúrgicos apenas $30,7 \%$ dos profissionais real izar am a higienização cor reta das mãos. E nquanto nos procedimentos clínicos o percentual foi $31,7 \%$. Constatou-se que, durante as cirurgias, os profissionais de nível superior realizaram melhor a higienização das mãos do que os de nível médio $(p<0,05)$ e, nos procedimentos clínicos, os profissionais de idade superior a 35 anos apresentaram melhor desempenho do que os mais moços $(p<0,05)$. Concluiu-se que menos de um terço dos profissionais observados executaram corretamente a higienização das mãos.

D escritores: Pessoal de saúde. Controle de infecções. Serviços de saúde. L avagem de mãos.

\section{RESUMEN}

El objeto del estudio ha sido determinar el estándar de la higienización de las manos de profesionales activos en U nidades Básicas de salud en diez municipios de la R egión Sur de Santa Catarina, B rasil, en 2008. Se trata de una investigación epidemiológica transversal, desarrollado con la observación de 369 profesionales de la salud. F ueron realizados test de asociación (Qui-cuadrado) control asociación de variables socio-demográficas y formación, nivel designificación 5\%. D urante procedimi entos qui rúrgi cos a penas $30,7 \%$ de los profesionales realizan la higienización cor recta delas manos. M ientras en los procedimientos clínicos el porcentajefuede $31,7 \%$. Con el test de asociación se constata queel nivel deformación se unió al desempeño de los profesional es que realizaron los procedimi entos quirúrgicos, mientras que los profesionales de edad superior a 35 años presentaron mejor desempeño en los procedimientos clínicos. Se concl uyó que menos de un tercio de los profesi onales observados estaba realizando cor rectamente la higienización de las manos.

D escriptores: Personal de salud. Control de infecciones. Servicios de la sal ud. L avado de manos.

Título: Calidad de la higienización de las manos de profesionales actuantes en unidades básicas de salud.

\section{ABST RACT}

The study aims to evaluethe quality of hand hygiene of professionals who worked in $\mathrm{B}$ asic $\mathrm{H}$ ealthcare $\mathrm{U}$ nits in ten cities in the south of thestate of Santa Catarina, B razil. T her research was conducted in 2008. It was a cross-sectional epidemi ological study that observed 369 health professionals. A chi-square test was carried out to verify the association of socio-demographic variables and training with the performance of the professionals. D uring the surgical procedures only $30.7 \%$ of the professionals performed a cor rect hand hygiene, whilein dinical procedures $31.7 \%$ did. D uring surgical procedure, graduated professionals did a better hand hygienethan professionals who only had high-school or equivalent training $(p<0.05)$, while professionals over 35 years old presented a better hygienein the clinical procedures than younger ones $(p<0.05)$. T he conclusion is that less than a third of the professionals were performing a proper hand hygiene

D escriptors: H ealth personnel. Infection control. H ealth services. H andwashing.

T itle: Quality of hand hygiene of active professionals in basic health units.

\footnotetext{
a Enfermeira, M estranda do Programa de Pós-G raduação em Ciências da Saúde da U niversidade do Sul de Santa Catarina (U NISU L), T ubarão, Santa Catarina, Brasil.

${ }^{\text {b }}$ D outora em Saúde Pública, Professora do Programa de Pós-Graduação em Saúde Pública da U niversidade Federal de Santa Catarina (UF SC), F lorianópolis, Santa Catarina, Brasil.

c M estre em Enfermagem, Professora do Curso de G raduação em Enfermagem da U NISU L, T ubarão, Santa Catarina, Brasil.

d D outora em O dontologia, Professora do Programa de Pós-G raduação em Ciências da Saúde da U N I SU L, T ubarão, Santa Catarina, Brasil.
} 


\section{INT RODUÇÃO}

Com o crescimento de novas formas e tipos de microorganismos, a preocupação com as infecções em locais de assistência à saúde se tornou um dos mais importantes problemas de saúde. Estas infecções são impactantes porque causam um aumento da resistência dos microrganismos aos antimicrobianos, uma prolongada incapacidade, uma sobrecarga financeira maciça, um alto custo para os pacientes e suas famílias, e as mortes em excesso(1).

As mãos dos profissionais da área da saúde servem como principal veículo de infecções cruzadas no ambiente hospitalar e demais locais de assistência à saúde. A microbiota das mãos consiste em microrganismos residentes e transitórios. Os microrganismos residentes são na maioria bactérias G ram-positivas tais como: Staphylococcus coagulase negativos, M icrococcus e al gumas espécies de corinebactérias ${ }^{(2,3)}$.

$\mathrm{N}$ a microbiota transitória, geralmente, predominam bactérias $\mathrm{G}$ ram-negativas, principalmente as enterobactérias, as do gênero $P$ seudomonas, bactérias aeróbicas formadoras de esporos, Staphylococcus aureus, fungos e vírus. Esses microorganismos apresentam maior patogenicidade, estando associados a surtos de infecção hospitalar (2-4).

Tem sido demonstrado que a higienização das mãos é a principal medida de inibição da disseminação de infecções em ambientes de assistência à saúde $^{(1,4,5)}$. A higienização engloba a: higienização simples, a degermação das mãos, a higienização antisséptica e a fricção antisséptica(2).

A deger mação das mãos é um procedimento dos profissionais de saúde no processo de remoção mecânica de microrganismos da superfície da pel ${ }^{(5)}$. Para realizar a mesma é necessário utilizar uma técnica específica, que consiste em: molhar as mãos com água, aplicar sabão, preferencialmente sobre a forma liquida, na quantidade necessária de produto para cobrir toda superfície das mãos. F azer movimentos de rotação das mãos, esfregando ambas as palmas e entrelaçar os dedos para cobrir toda superfície, friccionar os espaços interdigitais, as unhas e as pontas dos dedos, enxaguar as mãos em água corrente e secar com papel toalha. Ela deve ser executada antes e após os atendimentos aos pacientes ${ }^{(1)}$.
Em procedimentos cirúrgicos, o profissional de saúde deve realizar a higienização antisséptica, que consta na degermação das mãos e em seguida à utilização de um produto antisséptico na remoção de microorganismos, reduzindo a carga microbiana das mãos. Esta ação é indicada no atendimento aos pacientes portadores de microrganismos multi-resistentes, em casos de surtos, antes de qualquer procedimento cirúrgico e antes da realização de procedimentos invasivos ${ }^{(1,2,3)}$.

E ssa prática está em constante estudo em ambientes hospitalares, mostrando a adesão dos profissionais à higienização das mãos, a qualidade da microbiota das suas mãos, a necessidade da realização da ação e os produtos utilizados para maximizar o efeito da anti-sepsia das mãos ${ }^{(1,4-11)}$. 0 M inistério da Saúde recomenda como produto antisséptico o álcool a 70\%, a clorexidina, composto de iodo e o triclosan. A antissepsia só é eficaz quando as mãos não apresentam contaminações com fluidos orgânicos, material protéico nem sujida$\operatorname{des}^{(1-3)}$.

Em 2005, a Organização M undial da Saúde $(\mathrm{OMS})^{(1)}$ Iançou um desafio global para a melhoria dos serviços de saúde. 0 tópico escolhido para o primeiro desafio global para a segurança do paciente foi a infecção associada a assistência a saúde. A OM S considera que essas infecções ocorrem tanto em países desenvolvidos quanto naqueles em desenvolvimento. Este desafio foi intitulado "U ma assistência limpa é uma assistência mais segura". A ação chave nele prevista era promover a higienização das mãos na assistência a saúde em todo mundo. Foram escolhidos como locais-pilotos tanto hospitais modernos com alta tecnologia em países desenvolvidos quanto consultórios remotos em vilas de escassos recursos em países subdesenvolvidos. Os especial istas consideraram que a higienização das mãos é um problema vivido em hospitais, clínicas, sistemas de assistência à saúde, consultórios e postos de saúde.

No Brasil a maioria dos estudos é realizada nos hospitais. Assim, há a necessidade de investigações sobre a realização da higienização das mãos, em outros locais que realizam assistência à saúde. Por isso considerou-se importante determinar o padrão de higienização das mãos de profissionais que atuavam em U nidades Básicas de Saúde (UBS) avaliando a qualidade da degermação e da anti-sepsia 
das mãos antes e após procedimentos clínicos e cirúrgicos; os produtos mais utilizados na antisepsia; o local onde era realizada a higienização e a sua associação com fatores sócio-demográficos e formação profissional.

\section{MÉTODOS}

E ssa pesquisa caracteriza-se como um estudo epidemiológico transversal. É um corte de um estudo mais amplo, que aval iou as condições de biossegurança das U BS da Região Sul de Santa Catarina.

E sta região écomposta por 41 municípios, nos quais funcionavam 321 U BS. Foram selecionados os municípios que possuíam pelo menos 10 UBS, por viabilizar a coleta de dados, por uma questão logística.

Para o cálculo de amostra foi considerado a população descrita acima, com um nível de confiança de $95 \%$, um erro de $5 \%$ e uma prevalência de higienização das mãos de $40 \%$ (dado este inter mediário aquele apresentado pela Agência $\mathrm{N}$ acional de Vigilância Sanitária [ AN VISA ] ${ }^{(2)}$ obteve-se a amostra de 172 U BS. No entanto, participaram da pesquisa todas as 176 U BS em funcionamento nos municípios que obedeciam ao critério de seleção.

E m cada local foram observados todos os profissionais de saúde que estavam atuando no dia da visita do pesquisador e aceitaram participar, assinando o termo livre e esclarecido, totalizando 374 profissionais.

Os itens avaliados foram à higienização das mãos dos profissionais durantes procedimentos clínicos e cirúrgicos.

Considerou-se higienização correta para os procedimentos cirúrgicos à realização da técnica específica para degermação das mãos ${ }^{(2)}$, seguida do uso de um anti-séptico antes da colocação das luvas e a lavagem das mãos após a retirada das mesmas; a higienização correta para os procedimentos clínicos era obtida através da realização e técnica correta de degermação das mãos antes do procedimento clínico e a lavagem das mãos após o seu término(2).

As variáveis dependentes pesquisadas foram: degermação das mãos antes de procedimentos clínicos e de procedimentos cirúrgicos; uso de antisépticos após a degermação das mãos; os anti-sépticos utilizados; uso de pia específica para a deger- mação das mãos; utilização de técnica correta durante a degermação; lavagem das mãos após ambos os procedimentos. Foram investigadas, também, as seguintes variáveis independentes: tempo de formado, sexo, idade e categoria profissional.

A coleta dos dados foi realizada por 10 pesquisadores de campo, graduandos de odontologia, que for am submetidos a um treinamento. U ma professora do curso de enfermagem, participante da pesquisa, realizou a preparação dos acadêmicos e supervisionou a coleta de dados.

Os dados, coletados durante 0 ano de 2008, foram registrados em fichas apropriadas. Depois, foram inseridos em banco de dados construídos no programa $\mathrm{Exce}^{\circledR}$ e posteriormente transferido para o software Statistical Package for Social Sciences (SPSS) ${ }^{\circledR}$ versão 10.0 para W indows ${ }^{\circledR}$.

A análise foi conduzida por meio de estatística descritiva dos dados coletados. Foram realizados, também, testes de associação (qui-quadrado de Pearson) para verificar a associação de variáveis sócio-demográficas e formação, no desempenho da higienização das mãos realizadas pelos profissionais, utilizando-se o nível de confiança de 95\% ( $p<$ $0,05)$.

Para a realização deste estudo, foram respeitados os preceitos éticos, tendo sido aprovado pelo Comitê de Ética em Pesquisa da U niversidade do Sul de Santa Catarina, (Protocolo n06.410.4.06.III).

\section{RESULTADOS}

Foram observados 374 profissionais de saúde: 69 cirurgiões-dentistas, 69 auxiliares de consultório odontológico, 2 técnicos de higiene dental, 106 enfermeiros e 128 técnicos de enfermagem, que trabalhavam nas UBS avaliadas. Foram excluídos da pesquisa os médicos devido à ausência de atividade no momento da investigação e 5 profissionais, devido à ausência de dados na ficha de coleta.

Desta forma foram avaliados 369 profissionais. Estes pertenciam a uma faixa etária de 19 a 74 anos, com idade média de 35,1 anos (desvio padrão 10,6 anos), $84,83 \%$ eram do sexo feminino e $15,17 \%$ era do sexo masculino. 0 tempo de formado na profissão variou de um mês a 30 anos. D estes, $46,89 \%$ eram profissionais de nível superior e $53,11 \%$ profissionais de nível médio. 
Todos os 369 profissionais foram avaliados durante procedimentos clínicos. D estes profissionais, 176 também realizaram procedimentos cirúrgicos, sendo seus desempenhos novamente verificados e registrados (T abela 1 ).

Quando foi observada a higienização das mãos durante os procedimentos cirúrgicos o número de profissionais reduziu para 176, pois não foram todos os profissionais que realizaram este tipo de procedimento durantea visita dos pesquisadores de campo.

Os procedimentos foram avaliados de acordo com os seguintes critérios de higienização correta para os procedimentos cirúrgicos. Os dados coletados estão expressos na T abela 1.
Os profissionais observados que realizaram a anti-sepsia das mãos utilizaram os seguintes produtos: 0 álcool a $70 \%$ (por 53,4\%), a clorexidina ou o polivinil pirrolidona iodo (PVP-I) (por $7,9 \%$ ), um sabão ou sabonete com anti-séptico (por $22,1 \%$ ) e outros produtos por $16,5 \%$ dos profissionais.

A observação das U BS em relação ao local destinado para higienização das mãos evidenciou que somente $56,1 \%$ possuíam uma pia específica para os profissionais realizarem a higiene das mãos.

Os resultados dos testes de associação, realizados através do teste qui-quadrado de Pearson, estão expostos nas T abelas 2 e 3.

T abela 1 - Distribuição percentual da higienização das mãos em procedimentos clínicos e cirúrgicos realizados pelos profissionais das U nidades de Saúde da Região Sul de Santa Catarina, no ano de 2008.

\begin{tabular}{lcccc}
\hline Variáveis & \multicolumn{2}{c}{$\begin{array}{c}\text { Procedimentos clínicos } \\
\text { (N = 369) }\end{array}$} & \multicolumn{2}{c}{$\begin{array}{c}\text { Procedimentos cirúrgicos } \\
\text { (N = 176) }\end{array}$} \\
\cline { 2 - 5 } & $\mathbf{n}$ & \% & $\mathbf{n}$ & \% \\
\hline Higienização correta & 116 & 31,70 & 54 & 30,70 \\
$\begin{array}{l}\text { Degermação antes do } \\
\text { procedimento }\end{array}$ & 354 & 96,20 & 110 & 62,50 \\
Realização da anti-sepsia & 158 & 42,80 & 71 & 40,30 \\
$\begin{array}{l}\text { Degermação após 0 } \\
\text { procedimento }\end{array}$ & 350 & 94,90 & 167 & 94,90 \\
T écnica correta da & 216 & 58,50 & 103 & 58,50 \\
degermação & & & & \\
\hline
\end{tabular}

T abela 2 - Associação de variáveis sócio-demográficas e formação com a higienização das mãos realizada pelos profissionais que atuavam nas U nidades Básicas de Saúde da Região Sul de Santa Catarina, durante os procedimentos cirúrgicos, 2008.

\begin{tabular}{|c|c|c|c|c|c|}
\hline \multirow{2}{*}{$\begin{array}{l}\text { Variáveis } \\
\text { (N = 176) }\end{array}$} & \multicolumn{2}{|c|}{ Higienização correta } & \multicolumn{2}{|c|}{ Higienização incorreta } & \multirow{2}{*}{ Valor $p$} \\
\hline & $\mathrm{n}$ & $\%$ & $\mathbf{n}$ & $\%$ & \\
\hline \multicolumn{6}{|l|}{ Idade } \\
\hline A té 35 anos & 10 & 8,7 & 105 & 91,3 & - \\
\hline M ais 35 anos & 6 & 9,8 & 55 & 90,2 & 0,802 \\
\hline \multicolumn{6}{|l|}{ Sexo } \\
\hline M asculino & 4 & 11,8 & 30 & 88,2 & - \\
\hline Feminino & 12 & 8,5 & 130 & 91,5 & 0,546 \\
\hline \multicolumn{6}{|c|}{ Nível de formação } \\
\hline Superior & 12 & 13,8 & 75 & 86,2 & - \\
\hline M édio & 4 & 4,5 & 85 & 95,5 & $0,03 *$ \\
\hline \multicolumn{6}{|c|}{ T empo de formação } \\
\hline A té 5 anos & 7 & 7,3 & 99 & 92,7 & - \\
\hline$M$ ais 5 anos & 9 & 11,3 & 71 & 88,8 & 0,363 \\
\hline
\end{tabular}

$* p<0,05$. 
T abela 3 - Associação de al gumas variáveis com a higienização das mãos realizada pelos profissionais que atuavam nas U nidades Básicas de Saúde da Região Sul de Santa Catarina, durante os procedimentos clínicos, 2008.

\begin{tabular}{|c|c|c|c|c|c|}
\hline \multirow{2}{*}{$\begin{array}{l}\text { Variáveis } \\
\text { ( } N=176)\end{array}$} & \multicolumn{2}{|c|}{ Higienização correta } & \multicolumn{2}{|c|}{ Higienização incorreta } & \multirow{2}{*}{ Valor $\mathrm{p}$} \\
\hline & $n$ & $\%$ & $\mathbf{n}$ & $\%$ & \\
\hline \multicolumn{6}{|l|}{ Idade } \\
\hline A té 35 anos & 78 & 36,6 & 135 & 63,4 & - \\
\hline$M$ ais 35 anos & 74 & 47,4 & 82 & 52,6 & $0,03^{*}$ \\
\hline \multicolumn{6}{|l|}{ Sexo } \\
\hline M asculino & 29 & 51,8 & 27 & 48,2 & - \\
\hline Feminino & 123 & 39,3 & 190 & 60,7 & 0,08 \\
\hline \multicolumn{6}{|c|}{ N ível de formação } \\
\hline Superior & 74 & 42,8 & 99 & 57,2 & - \\
\hline M édio & 78 & 39,8 & 118 & 60,2 & 0,562 \\
\hline \multicolumn{6}{|c|}{ T empo de formação } \\
\hline A té 5 anos & 76 & 41,3 & 108 & 58,7 & - \\
\hline M ais 5 anos & 76 & 41,1 & 109 & 58,9 & 0,965 \\
\hline
\end{tabular}

\section{DISCUSSÃO}

Durante a rotina dos serviços de saúde a higienização das mãos deve ser realizada corretamente independente da técnica ou procedimento que o profissional executará a seguir. N este estudo não foi o observado. A penas 30,7\% dos profissionais, durante os procedimentos cirúrgicos, e 31,7\% nos procedimentos clínicos realizaram-na de acordo com as normas preconizadas pela Agência $\mathrm{Na}$ cional de Vigilância Sanitária (AN VISA) (2).

E ntretanto o número de profissionais que realizou a degermação das mãos antes dos procedimentos foi maior (62,5\% e $96,2 \%$, respectivamente). Chama a atenção que a degermação das mãos após os procedimentos foi realizado pela grande maioria dos profissionais, $94,9 \%$ dos procedimentos clínicos e cirúrgicos.

Quando observados a realização da técnica correta de degermação, o número de acerto caiu para $58,5 \%$. E ste fato pode ter ocorrido, dentre outros fatores, pela pressa que os profissionais têm ao fazê-la entre os atendimentos, pela falta de profissionais para atender uma alta demanda de pacientes, pelo alto número de oportunidades que exigem a higienização das mãos durante a rotina destes profissionais ${ }^{(1)}$. A técnica exige minúcias que são frequentemente deixadas de lado quando o procedimento é feito rapidamente. No caso particular das U nidades Básicas de Saúde a quantidade de pacientes que devem ser atendidos é grande e exi- ge que os profissionais executem suas tarefas agilmente. E ste fato, porém, não justifica a negligência no procedimento sabidamente essencial no controle das infecções cruzadas $^{(1-3)}$.

0 desempenho dos profissionais de nível superior, cirurgiões-dentistas e enfermeiros, foi meIhor do que 0 das equipes auxiliares $(p=0,03)$ apenas no preparo das mãos antes das cirurgias (T abela 2). A pesar disso o desempenho dos profissionais de nível superior deixou muito a desejar, pois somente 13,8\% estavam realizando a higienização correta.

Resultados semelhantes foram encontrados em uma pesquisa realizada em uma U nidade de ter apia intensiva (UTI), que teve por objetivo avaliar o cumprimento da técnica da lavagem das mãos em procedimentos invasivos e não invasivos. Ficou constatado que somente $50 \%$ dos profissionais da saúde realizaram a higienização das mãos antes de procedimentos invasivos e $44 \%$ nos procedimentos não invasivos ${ }^{(8)}$.

A observação de 125 profissionais de diversas categorias ${ }^{(8)}$ também, demonstrou que a prática da degermação era negligenciada, pois só $64 \%$ a realizavam assim que entravam no local. Entretanto, outro estudo(12), também realizado em UTI, demonstrou melhores resultados, pois $75,9 \%$ dos enfermeiros e $57,2 \%$ dos técnicos de enfermagem realizavam corretamente a higienização das mãos. E sses estudos colocam em evidência um percentual muito al to de fal has. 
Outra variável investigada foi a idade que apresentou associação com a qualidade da higienização das mãos durante procedimentos clínicos, sendo que $47,4 \%$ dos profissionais com mais de 35 anos de idade realizaram-na corretamente, enquanto este percentual baixou para $36,6 \%$ quando os profissionais eram mais jovens (T abela 3). N ão há referencias em literatura que demonstram resultados semel hantes ou controversos, o que pode ser atribuído são fatores de não adesão a higienização, como: baixo risco de adquirir infecções devido a idade, prioridade o paciente, falta de conhecimento e ressecamento e irritação da pele das mãos ${ }^{(2)}$.

Os demais fatores avaliados não mostraram associação com a qualidade da higienização das mãos dos profissionais (T abelas 2 e 3 ).

0 uso adequado dos anti-sépticos no preparo das mãos para os procedimentos cirúrgicos, nos quais a anti-sepsia das mãos é indispensável, também, foi deficiente não sendo realizado por mais da metade dos profissionais $(59,7 \%)$ envolvidos nesta pesquisa (T abela 1). No entanto aqueles que os utilizaram, optaram por produtos que estavam de acordo com o que é preconizado pelo $M$ inistério da Saúde( ${ }^{(2,3)}$. 0 álcool a $70 \%$ foi o produto mais utilizado, sendo empregado por 53,4\% dos profissionais que realizaram anti-sepsia. A baixa adesão ao uso dos anti-sépticos quando da preparação das mãos para os procedimentos cirúrgicos $(40,3 \%)$ pode ter sido decorrente da ação irritante desses produtos para a pele das mãos. Por isso, os profissionais que prestam assistência à saúde devem ser orientados sobre a real ização de testes pilotos para escolher 0 anti-séptico que irá usar ${ }^{(1)}$.

Outro detal he interessante encontrado neste estudo foi à adesão dos profissionais à lavagem das mãos após os atendimentos dos pacientes. Dentre eles $94,9 \%$ realizavam-na. Corroborando com este achado, um estudo colocou em evidência que os enfermeiros de uma unidade de terapia intensiva realizavam mais frequentemente a higienização das mãos após os procedimentos. Das 1246 observações realizadas, $87 \%$ da ação ocorriam ao término de cada atividade ${ }^{(13)}$. U ma explicação provável é que 0 uso das luvas de látex deixa resíduos de tal co que causam desconfor to e estimulam a lavagem das mãos.

Para que a higienização das mãos seja realizada na forma correta, a existência de um lavatório ou uma pia destinada somente a higienização das mãos é um objeto essencial(1). N os campos de coleta apenas $56,9 \%$ possuíam uma pia adequada para realizar a ação. Sempre que houver pacientes, manipulações, medicamentos, é obrigatória a provisão de recursos para higienização das mãos, dentre el es uma pia exclusiva para ação(2). A credita-se que o baixo percentual apresentado nas U BS avaliadas, seja em função da falta de informação em relação a esse quesito. Espera-se que à medida que trabalhos nesta perspectiva sejam realizados, ocorra o estimulo para a adoção desta importante medida para o controle de infecções cruzadas em todas as U BS brasileiras, chegando-se ao ideal alcançado por uma unidade hospitalar de N ova York, na qual $100 \%$ dos ambientes possuíam uma pia específica para higiene das mãos ${ }^{(13)}$.

E $m$ unidades básicas de saúde todo grupo de trabalho possui um profissional de nível superior, que realiza uma supervisão do trabal ho desempenhado pelos profissionais de nível médio. Portanto os técnicos de enfermagem tinham como supervisor um enfermeiro, enquanto que os auxiliares de consultórios odontológicos e técnicos de higiene oral trabal havam sob a supervisão de um cirurgiãodentista. Desta maneira esta avaliação do desempenho dos profissionais das UBS colocou em evidência não só a falta de adesão dos profissionais graduados, como também ineficiência das supervisões quanto ao procedimento da higienização das mãos.

É necessário que haja um maior cuidado dos profissionais que atuam nas USB para que realizem a higienização das mãos corretamente. Para tal, sugere-se que sejam efetuadas capacitações periódicas destes profissionais e os gestores tenham um maior empenho no cumprimento das normas determinadas pela AN VISA ${ }^{(2)}$. A final, os profissionais ao observarem o que está preconizado nestas normas estarão zelando não só pela saúde da população por eles atendida, como por sua própria saúde.

Considera-se importante que estudos semeIhantes sejam realizados em outras regiões brasileiras para mapear a realidade das condições de trabalho das U BS nacionais.

\section{CONCLUSÕES}

A investigação, que avaliou a higienização das mãos realizadas pelos profissionais de saúde atuantes em U BS, mostrou que: 
a) apenas aproximadamente um terço dos profissionais observados realizou a higienização correta das mãos durante os procedimentos clínicos e cirúrgicos;

b) o término dos procedimentos foi o momento de realização mais frequente da higienização das mãos;

c) um número expressivo de profissionais $(43,9 \%)$ não tinha acesso a uma pia próprio para lavagem das mãos;

d) 0 nível de formação dos profissionais estava associado à higienização das mãos em procedimentos cirúrgicos e a variável idade à higienização nos procedimentos clínicos.

\section{REFERÊNCIAS}

1 W orld H ealth Organization. W HO guidelines on hand hygiene in health care: a Summary. G eneva; 2005.

2 M inistério da Saúde (BR ), A gência N acional de Vigilância Sanitária. Segurança do paciente: higienização das mãos. Brasília (D F ); 2007.

3 M inistério da Saúde (BR), A gência N acional de Vigilância Sanitária. Serviços odontológicos: prevenção e controle de riscos. Brasília (DF); 2006.

4 Pal os M AP, Silva DVB, Gir E, Canini SR M S, Anders $P S$, L eão LSN O, et al. M icrobiota das mãos de mães e de profissionais de saúde de uma maternidade de G oiânia. Rev E letrônica E nferm [ I nternet] . 2009 [ citado 2010 ago 18];11(3):573-8. Disponível em: http:/ / www.fen.ufg.br/ revista/ v11/n3/ v11n3a14.htm.

5 Kampf G, L öffler H, Gastmeier P. H and hygiene for the prevention of nosocomial infections. Dtsch Arztebl Int. 2009;106(40):649-55.

\section{Endereço da autora / Dirección del autor / Author's address:}

Ana Claudina Predêncio Serratine Universidade do Sul de Santa Catarina

D epartamento de Pós-G raduação em Ciências da Saúde

Av. José A cácio M oreira, 787, D ehon

88704-900, T ubarão, SC

E-mail:anaclaudina@unisul.br
6 Boyce JM . Antiseptic technology: access, affordability and acceptance. E merg Infect D is. 2001;7(2):231-3.

7 Pittet D, M ourouga P, Perneger T V. Compliance with handw ashing in a teaching hospital. A nn I ntern M ed. 1999;130:126-30.

8 Pereira LS, Souza ACS, Tipple AFV, Pereira M S, Junqueira ALN. Anti-sepsia para administração de medicamentos por via endovenosa e intramuscular. Rev Eletrônica E nferm [ Internet] . 2006 [ citado 2010 set 05] ;8(1):75-82. Disponível em: http:/ / www.fen. ufg.br/ revista/revista8_1/ original_10.htm.

$9 \mathrm{M}$ endonça AP, Fernandes M SC, Azevedo JM R, SiIveira W CRS, Souza ACS. Lavagem das mãos: adesão dos profissionais de saúde em uma unidade de terapia intensiva neonatal. A cta Sci. Health Sci. 2003;25(2): 147-53.

10 M artinez M R, Campos LAAF, N ogueira PCK. A desão à técnica de lavagem de mãos em U nidade de Terapia I ntensiva N eonatal. Rev Paul Pediatr. 2009; 27(2):179-85.

11 Cruz EDA, Pimenta F C, Palos M AP, Silva SR M , G ir E. Higienização de mãos: 20 anos de divergências entre a prática e o idealizado. Cienc Enferm. 2009; 15(1):33-8.

12 N eves ZCP, T ipple AFV, Souza ACS, Pereira M S, $M$ elo DS. $H$ and hygiene: the impact of incentive strategies on adherence among healthcare workers from a new bor $n$ intensive care unit. R ev $L$ atino- $A m$ Enfermagem 2006;14(4):546-52.

13 O'Boyle CA, H enly SJ, Larson E. U nderstanding adherence to hand hygiene recommendations: the theory of planned behavior. A m J I nfect Control. 2001;29(6):352-60.

Recebido em: 08/ 11/ 2010

A provado em: 26/07/ 2011 